\title{
Analysis of a Periodic Single Species Population Model Involving Constant Impulsive Perturbation
}

\author{
Ronghua Tan, ${ }^{1,2}$ Zuxiong Li, ${ }^{1,2}$ Shengliang Guo, ${ }^{1,2}$ and Zhijun Liu ${ }^{1,2}$ \\ ${ }^{1}$ Key Laboratory of Biologic Resources Protection and Utilization of Hubei Province, \\ Hubei University for Nationalities, Enshi, Hubei 445000, China \\ ${ }^{2}$ Department of Mathematics, Hubei University for Nationalities, Enshi, Hubei 445000, China
}

Correspondence should be addressed to Zhijun Liu; zhijun_liu47@hotmail.com

Received 11 January 2014; Accepted 22 May 2014; Published 12 June 2014

Academic Editor: Sabri Arik

Copyright (C) 2014 Ronghua Tan et al. This is an open access article distributed under the Creative Commons Attribution License, which permits unrestricted use, distribution, and reproduction in any medium, provided the original work is properly cited.

\begin{abstract}
This is a continuation of the work of Tan et al. (2012). In this paper a periodic single species model controlled by constant impulsive perturbation is investigated. The constant impulse is realized at fixed moments of time. With the help of the comparison theorem of impulsive differential equations and Lyapunov functions, sufficient conditions for the permanence and global attractivity are established, respectively. Also, by comparing the above results with corresponding known results of Tan et al. (2012) (i.e., the above model with linear impulsive perturbations), we find that the two different types of impulsive perturbations have influence on the above dynamics. Numerical simulations are presented to substantiate our analytical results.
\end{abstract}

\section{Introduction}

It is well known that many mathematical models can be expressed by impulsive differential equations, and in recent years many impulsive differential equations have been introduced in population dynamics and neural networks, such as harvesting or stocking [1-4], chemostat [5], vaccination [6], integrated pest management [7], chemotherapeutic treatment of disease [8], and neural networks [9]. Also, there has been a significant development in theory of impulsive differential equation. For example, [10-17] studied the existence and stability of systems. In this paper, we investigate the permanence and global attractivity of a periodic impulsive system (see (7a) and (7b)).

In 1978, Ludwig et al. [18] investigated the asymptotic behavior of the following single species autonomous system:

$$
y^{\prime}(t)=y(t)[a-b y(t)]-E(y),
$$

where $y(t)$ is the density of species $y$ at time $t, a$ is the intrinsic growth rate, $b$ is the self-inhibition rate, and $E(y)$-term represents predation. Predation is an increasing function and usually saturates for large enough $y$. The predation term $E(y)$ drops rapidly if the density of species $y$ is small. To be specific
Murray [19] took the form for $E(y)$ suggested by Ludwig et al. [18], namely, $\alpha y^{2}(t) /\left(\beta+y^{2}(t)\right)$, and the dynamics of $y(t)$ is then governed by

$$
y^{\prime}(t)=y(t)[a-b y(t)]-\frac{\alpha y^{2}(t)}{\beta+y^{2}(t)}
$$

where the positive coefficients $\alpha$ and $\beta$ are measures of saturation value. Murray [19] discussed the existence and stability of equilibria of system (2).

To investigate the effects of other specific forms of $E(y)$ on system (1), similar to Murry [19], in [1] we took $E(y)=$ $c y(t) /(d+y(t))$ and established the following single species system:

$$
y^{\prime}(t)=y(t)[a-b y(t)]-\frac{c y(t)}{d+y(t)} .
$$

Obviously, $E(y)=c y(t) /(d+y(t))$ is an increasing function with respect to $y$ and has a saturation value, and the positive coefficients $c$ and $d$ are measures of saturation value. In [1], we further consider the possible effects of periodically varying environment and impulsive perturbations on system 
(3) and established the following periodic system with linear impulsive perturbations:

$$
\begin{gathered}
y^{\prime}(t)=y(t)[a(t)-b(t) y(t)]-\frac{c(t) y(t)}{d(t)+y(t)}, \\
t \neq \tau_{k}, \quad k \in \mathbf{N}, \\
\Delta y\left(\tau_{k}\right)=I\left(y\left(\tau_{k}\right)\right)=\gamma_{k} y\left(\tau_{k}\right),
\end{gathered}
$$

where $y(0)>0, \Delta y\left(\tau_{k}\right)=y\left(\tau_{k}^{+}\right)-y\left(\tau_{k}\right), \tau_{k}$ is an impulsive point for every $k$, and $0<\tau_{1}<\tau_{2}<\cdots<$ $\tau_{k}<\cdots$ and $\mathbf{N}$ is the set of positive integers. $b(t), c(t)$, and $d(t)$ are positive continuous $T$-periodic functions. The intrinsic growth rate $a(t)$ is not necessarily positive, since the environment fluctuates randomly; in a bad environment $a(t)$ may be negative when the birth rate is smaller than the death rate. $\left\{\gamma_{k}\right\}$ is a real sequence and there exists an integer $q>0$ such that $\gamma_{k+q}=\gamma_{k}, \tau_{k+q}=\tau_{k}+T$. We have investigated the permanence and global attractivity of system (4a) and (4b) and two results can be redescribed as follows.

Result A (see Lemma 3.2 in [1]). If assumption

$$
\prod_{k=1}^{q}\left(1+\gamma_{k}\right) \exp \left\{\int_{0}^{T}\left[a(t)-\frac{c(t)}{d(t)} d t\right]\right\}>1
$$

holds, then system (4a) and (4b) is permanent; that is, there exist positive constants $m, M$, and $\widetilde{t}$ such that any positive solution $y(t)$ of system (4a) and (4b) satisfies $m \leq y(t) \leq M$, for $t \geq \tilde{t}$.

Result $B$ (see Theorem 3.1 in [1]). In addition to (5), assume further that

$$
b(t)>\frac{c(t)}{(d(t)+m)^{2}} .
$$

Then, system (4a) and (4b) is global attractive, where $m$ is described as Result A.

In this contribution, we continue to consider system (4a) with constant impulsive perturbation (i.e., $I\left(y\left(\tau_{k}\right)\right)=p$ ) and obtain the following system:

$$
\begin{gathered}
y^{\prime}(t)=y(t)[a(t)-b(t) y(t)]-\frac{c(t) y(t)}{d(t)+y(t)}, \\
t \neq \tau_{k}, \quad k \in \mathbf{N}, \\
\Delta y\left(\tau_{k}\right)=I\left(y\left(\tau_{k}\right)\right)=p .
\end{gathered}
$$

The positive constant $p$ is the increased amount of species $y$ at $\tau_{k}$ which implies that species $y$ is subjected to impulsive increase at a constant rate $p$.

In this paper, our main purpose is to establish sufficient conditions for the permanence and global attractivity of system (7a) and (7b) and compare them with the effects of the above two different types of impulsive perturbations on the above dynamics. The organization of this paper is as follows. In Section 2, we present some preliminary lemmas.
In Section 3, two main results on the permanence and global attractivity of system (7a) and (7b) are presented. In Section 4 , some examples together with their corresponding numerical simulations are presented to verify the validity of the proposed criteria.

\section{Preliminaries}

The following Lemmas 1 and 2 are useful for establishing the permanence of system (7a) and (7b). Lemma 1 shows that the Malthus growth model with constant impulse has a good asymptotic behavior and a detailed proof can be seen in Lemma 2.3 in [4].

Lemma 1 (see $[2,4])$. Consider the following impulsive system:

$$
\begin{gathered}
x^{\prime}(t)=a(t) x(t), \quad t \neq \tau_{k}, k \in \mathbf{N}, \\
x\left(\tau_{k}^{+}\right)=x\left(\tau_{k}\right)+p,
\end{gathered}
$$

where $x(0)>0, a(t)$ is a continuous $T$-periodic function with $\int_{0}^{T} a(t) d t<0$, the constant $p>0$, and there is an integer $q>0$ such that $\tau_{k+q}=\tau_{k}+T, k \in \mathbf{N}$. System (8) has a unique positive $T$-periodic solution which is globally asymptotically stable.

Lemma 2 (see [4]). Consider the following impulsive single species system:

$$
\begin{gathered}
x^{\prime}(t)=x(t)[a(t)-b(t) x(t)], \quad t \neq \tau_{k}, k \in \mathbf{N}, \\
x\left(\tau_{k}^{+}\right)=x\left(\tau_{k}\right)+p,
\end{gathered}
$$

where $x(0)>0, a(t)$ and $b(t)$ are continuous T-periodic functions, and $b(t)>0$, the constant $p>0$, and there exists an integer $q>0$ such that $\tau_{k+q}=\tau_{k}+T$. Then,

(1) species $x$ is permanent;

(2) any two positives $x(t)$ and $\bar{x}(t)$ satisfy $\lim _{t \rightarrow+\infty} \mid x(t)-$ $\bar{x}(t) \mid=0$.

The following Lemma 3 is useful for proving the global attractivity of system (7a) and (7b). We first give the following notations and spaces of functions.

Let $\mathbf{J} \subset \mathbf{R}=(-\infty,+\infty)$ and $\mathbf{J}=\left[\tau_{0},+\infty\right) . P C(\mathbf{J}, \mathbf{R})=\{x:$ $\mathbf{J} \rightarrow \mathbf{R}: x$ is continuous for $t \in \mathbf{J}, t \neq \tau_{k}$, continuous from the left for $t \in \mathbf{J}$ and discontinuous of the first kind occurring at the point $\left.\tau_{k} \in \mathbf{J}, k \in \mathbf{N}\right\}$, and $P C^{1}(\mathbf{J}, \mathbf{R})=\{x \in P C(\mathbf{J}, \mathbf{R}): x$ is continuously differential for $t \in \mathbf{J}, t \neq \tau_{k}, x^{\prime}\left(\tau_{k}^{+}\right)$and $x^{\prime}\left(\tau_{k}^{-}\right)$ exist, $k \in \mathbf{N}$.

Lemma 3 (see [20]). If the function $u \in P C^{1}\left(\left[\tau_{0},+\infty\right), \mathbf{R}\right)$ satisfies the inequalities

$$
\begin{gathered}
x^{\prime}(t) \leq f(t) x(t)+\alpha(t), \quad t \neq \tau_{k}, t \geq t_{0}, \\
x\left(\tau_{k}^{+}\right) \leq d_{k} x\left(\tau_{k}\right)+\beta_{k}, \quad k \in \mathbf{N},
\end{gathered}
$$


where $f(t), \alpha(t) \in P C\left(\left[\tau_{0},+\infty\right), \mathbf{R}\right), d_{k}>0$, and $\beta_{k}$ are constants for $k \in \mathbf{N}$, then, for $t \geq \tau_{0}$,

$$
\begin{aligned}
x(t) \leq & x\left(\tau_{0}\right) \prod_{\tau_{0}<\tau_{k}<t} d_{k} \exp \left(\int_{\tau_{0}}^{t} f(s) d s\right) \\
& +\sum_{\tau_{0}<\tau_{k}<t}\left(\prod_{\tau_{k}<t_{j}<t} d_{j} \exp \left(\int_{\tau_{k}}^{t} f(s) d s\right)\right) \beta_{k} \\
& +\int_{\tau_{0}}^{t} \prod_{s<\tau_{k}<t} d_{k} \exp \left(\int_{s}^{t} f(r) d r\right) \alpha(s) d s .
\end{aligned}
$$

Analogously, we have

$$
\begin{aligned}
x(t) \geq & x\left(\tau_{0}\right) \prod_{\tau_{0}<\tau_{k}<t} d_{k} \exp \left(\int_{\tau_{0}}^{t} f(s) d s\right) \\
& +\sum_{\tau_{0}<\tau_{k}<t}\left(\prod_{\tau_{k}<t_{j}<t} d_{j} \exp \left(\int_{\tau_{k}}^{t} f(s) d s\right)\right) \beta_{k} \\
& +\int_{\tau_{0}}^{t} \prod_{s<\tau_{k}<t} d_{k} \exp \left(\int_{s}^{t} f(r) d r\right) \alpha(s) d s
\end{aligned}
$$

for $t \geq \tau_{0}$ if all the inequalities of (10) are inverse.

\section{Permanence and Global Attractivity}

We first give the main result on the permanence of system (7a) and (7b).

Theorem 4. System (7a) and (7b) is permanent; that is, there exist positive constants $h, H$, and $t^{*}$ such that any positive solution $y(t)$ of system (7a) and (7b) satisfies $h \leq y(t) \leq H$ for $t \geq t^{*}$.

Proof. It follows from (7a) and (7b) that we obtain

$$
\begin{array}{r}
y(t)=y\left(\tau_{k-1}^{+}\right) \\
\times \exp \left\{\int_{\tau_{k-1}}^{t}\left[a(s)-b(s) y(s)-\frac{c(s)}{d(s)+y(s)}\right] d s\right\}, \\
t \in\left(\tau_{k-1}, \tau_{k}\right], \\
y\left(\tau_{k}^{+}\right)=y\left(\tau_{k}\right)+p .
\end{array}
$$

Obviously, if $y\left(0^{+}\right)=y(0)>0$, then $y(t)>0$ for $t>0$.

We first show that any positive solution $y(t)$ of system (7a) and $(7 \mathrm{~b})$ is uniformly ultimately upper bounded. To do this, we have from $(7 a)$ and $(7 b)$ that

$$
\begin{gathered}
y^{\prime}(t) \leq y(t)[a(t)-b(t) y(t)], \quad t \neq \tau_{k}, k \in \mathbf{N}, \\
y\left(\tau_{k}^{+}\right)=y\left(\tau_{k}\right)+p .
\end{gathered}
$$

Consider the comparison system of (14):

$$
\begin{gathered}
w^{\prime}(t)=w(t)[a(t)-b(t) w(t)], \quad t \neq \tau_{k}, k \in \mathbf{N}, \\
w\left(\tau_{k}^{+}\right)=w\left(\tau_{k}\right)+p .
\end{gathered}
$$

By (1) in Lemma 2 we know that system (15) is permanent, which implies that there exist constants $H>0$ and $\bar{t}>0$ such that

$$
w(t) \leq H, \quad \text { for } t \geq \bar{t} .
$$

Let $w(t)$ be the solution of (15) with $w(0)=y(0)$. By the comparison theorem of impulsive differential equations and (16), we obtain

$$
y(t) \leq H, \quad \text { for } t \geq \bar{t} .
$$

Next, we prove that $y(t)$ is uniformly ultimately lower bounded. It follows from (7a), (7b), and (17) that we have, for $t \geq \bar{t}$

$$
\begin{gathered}
y^{\prime}(t) \geq y(t)\left[a(t)-b(t) H-\frac{c(t)}{d(t)}\right] \\
\geq y(t)\left[a(t)-b(t) H-\frac{c(t)}{d(t)}-\varrho\right], \quad t \neq \tau_{k}, \\
y\left(\tau_{k}^{+}\right)=y\left(\tau_{k}\right)+p
\end{gathered}
$$

where the positive constant $\varrho$ satisfies

$$
\int_{0}^{T}\left[a(t)-b(t) H-\frac{c(t)}{d(t)}-\varrho\right] d t<0 .
$$

It follows from Lemma 1 that the comparison system of (18)

$$
\begin{gathered}
v^{\prime}(t)=v(t)\left[a(t)-b(t) H-\frac{c(t)}{d(t)}-\varrho\right], \\
t \neq \tau_{k}, \quad k \in \mathbf{N}, \\
v\left(\tau_{k}^{+}\right)=v\left(\tau_{k}\right)+p
\end{gathered}
$$

has a unique positive, globally asymptotically stable $T$ periodic solution, denoted by $\widehat{v}(t)$. The asymptotic property of $\widehat{v}(t)$ implies that there exist $t^{*} \geq \bar{t}$ and $\epsilon>0$ such that

$$
v(t) \geq \widehat{v}(t)-\epsilon, \quad \text { for } t \geq t^{*},
$$

where the constant $\epsilon<(1 / 2) \min _{t \in[0, T]}\{\hat{v}(t)\}$. Let $v(t)$ be the solution of (20) with $v\left(t^{*}\right)=y\left(t^{*}\right)$. By the comparison theorem of impulsive differential equations and (18)-(21), we have

$$
y(t) \geq \widehat{v}(t)-\epsilon \geq \min _{t \in[0, T]}\{\widehat{v}(t)\}-\epsilon=h, \quad \text { for } t \geq t^{*} .
$$

From (17) and (22), one has

$$
h \leq y(t) \leq H, \quad \text { for } t \geq t^{*} .
$$

The proof of Theorem 4 is complete.

Remark 5. Theorem 4 shows that the permanence of system (7a) and (7b) is irrespective of the size of the positive impulse $p$.

In the following, we continue to investigate the global attractivity of system (7a) and (7b). 
Theorem 6. If system (7a) and (7b) satisfies

$$
\begin{gathered}
b(t)>\frac{c(t)}{(d(t)+h)^{2}}, \\
h+p=H,
\end{gathered}
$$

then system (7a) and (7b) is global attractive, where $h$ and $H$ are described as in Theorem 4.

Proof. Let $y^{*}(t)$ and $y(t)$ be any two positive solutions of system (7a) and (7b). By Theorem 4, we know that there exists a $\hat{t}$ large enough such that, for all $t>\widehat{t}>t^{*}$,

$$
h \leq y^{*}(t) \leq H, \quad h \leq y(t) \leq H .
$$

Consider a Lyapunov functional $V(t)$ defined by

$$
V(t)=\left|\ln y^{*}(t)-\ln y(t)\right| .
$$

Calculating and estimating the upper right derivative $D^{+} V(t)$ of $V(t)$ along the solutions of (7a) and (7b), we get, for $t>\widehat{t}$ and $t \neq \tau_{k}$, that

$$
\begin{aligned}
D^{+} V(t)= & \operatorname{sgn}\left(y^{*}(t)-y(t)\right)\left(\frac{y^{* \prime}(t)}{y^{*}(t)}-\frac{y^{\prime}(t)}{y(t)}\right) \\
= & \operatorname{sgn}\left(y^{*}(t)-y(t)\right) \\
& \times\left(-b(t)\left(y^{*}(t)-y(t)\right)\right. \\
& \left.\quad+\frac{c(t)\left(y^{*}(t)-y(t)\right)}{\left(d(t)+y^{*}(t)\right)(d(t)+y(t))}\right) \\
\leq & -\left[b(t)-\frac{c(t)}{(d(t)+h)^{2}}\right]\left|y^{*}(t)-y(t)\right| .
\end{aligned}
$$

From assumption (24), we obtain that there exists a constant $\lambda>0$ such that

$$
b(t)>\frac{c(t)}{(d(t)+h)^{2}}+\lambda,
$$

which, together with (28), yields

$$
D^{+} V(t) \leq-\lambda\left|y^{*}(t)-y(t)\right| \text {. }
$$

By the mean value theorem and (26), for any closed interval contained in $t \in\left(\tau_{k}, \tau_{k+1}\right], k=m, m+1, \ldots$, and $\tau_{m}>\widehat{t}$, one has

$$
\frac{1}{H}\left|y^{*}(t)-y(t)\right| \leq\left|\ln y^{*}(t)-\ln y(t)\right| \leq \frac{1}{h}\left|y^{*}(t)-y(t)\right| .
$$

Therefore, for $t \in\left(\tau_{k}, \tau_{k+1}\right], k=m, m+1, \ldots$, and $\tau_{m}>\widehat{t}$, from (30) and (31), we have

$$
\begin{aligned}
D^{+} V(t) & \leq-\lambda\left|y^{*}(t)-y(t)\right| \\
& \leq-\lambda h\left|\ln y^{*}(t)-\ln y(t)\right|=-\psi V(t),
\end{aligned}
$$

where $\psi=\lambda h>0$.
On the other hand, for $t=\tau_{k}, k \in \mathbf{N}$, we have

$$
\begin{aligned}
V\left(\tau_{k}^{+}\right) & =\left|\ln \left(y^{*}\left(\tau_{k}\right)+p\right)-\ln \left(y\left(\tau_{k}\right)+p\right)\right| \\
& =\left|g^{\prime}(z)\right|_{z=\mu_{k}}\left(y^{*}\left(\tau_{k}\right)-y\left(\tau_{k}\right)\right) \mid \\
& =\frac{1}{\mu_{k}+p}\left|y^{*}\left(\tau_{k}\right)-y\left(\tau_{k}\right)\right|,
\end{aligned}
$$

where $\mu_{k}$ is between $y^{*}\left(\tau_{k}\right)$ and $y\left(\tau_{k}\right)$ and $g(z)=\ln (z+p)$.

It follows from (26) and (33) that, for $\tau_{k}>\widehat{t}, k=m, m+$ $1, \ldots$,

$$
V\left(\tau_{k}^{+}\right) \leq \frac{1}{h+p}\left|y^{*}\left(\tau_{k}\right)-y\left(\tau_{k}\right)\right|
$$

Again, by the mean value theorem, we have

$$
\begin{aligned}
\left|\ln y^{*}\left(\tau_{k}\right)-\ln y\left(\tau_{k}\right)\right| & =\left|r^{\prime}(\sigma)\right|_{\sigma=\eta_{k}}\left(y^{*}\left(\tau_{k}\right)-y\left(\tau_{k}\right)\right) \mid \\
& =\frac{1}{\eta_{k}}\left|y^{*}\left(\tau_{k}\right)-y\left(\tau_{k}\right)\right|
\end{aligned}
$$

where $\eta_{k}$ is between $y^{*}\left(\tau_{k}\right)$ and $y\left(\tau_{k}\right)$ and $r(\sigma)=\ln (\sigma)$.

Furthermore, we have from (26), (34), and (35) that, for $\tau_{k}>\widehat{t}_{2}$,

$$
\begin{aligned}
\frac{1}{h+p}\left|y^{*}\left(\tau_{k}\right)-y\left(\tau_{k}\right)\right| & \leq \frac{H}{h+p}\left|\ln y^{*}\left(\tau_{k}\right)-\ln y\left(\tau_{k}\right)\right| \\
& =\mathscr{H} V\left(\tau_{k}\right),
\end{aligned}
$$

where

$$
\mathscr{H}=\frac{H}{h+p} .
$$

Recall assumption (25); we obtain that $\mathscr{H}=1$; thus, we have from (34)-(36) that

$$
V\left(\tau_{k}^{+}\right) \leq V\left(\tau_{k}\right) .
$$

Combining (32) and (38), we have

$$
\begin{gathered}
D^{+} V(t) \leq-\psi V(t), \quad t \neq \tau_{k}, t>\widehat{t}, \\
V\left(\tau_{k}^{+}\right) \leq V\left(\tau_{k}\right), \quad \tau_{k}>\widehat{t}, k=m, m+1, \ldots
\end{gathered}
$$

Note that $\tau_{m}$ is an impulsive point; by Lemma 3, we obtain that, for $t>\tau_{m}>\widehat{t}$,

$$
V(t) \leq V\left(\tau_{m}^{+}\right) \exp \left(\int_{\tau_{m}}^{t}-\psi d s\right)=V\left(\tau_{m}^{+}\right) \exp \left(-\psi\left(t-\tau_{m}\right)\right) .
$$

Obviously, it follows from (26) and (27) that

$$
\begin{aligned}
V\left(\tau_{m}^{+}\right) & =\left|\ln \left(y^{*}\left(\tau_{m}\right)+p\right)-\ln \left(y\left(\tau_{m}\right)+p\right)\right| \\
& \leq \max \{2|\ln (h+p)|, 2|\ln (H+p)|\},
\end{aligned}
$$

which implies that $V\left(\tau_{m}^{+}\right)$is bounded. 


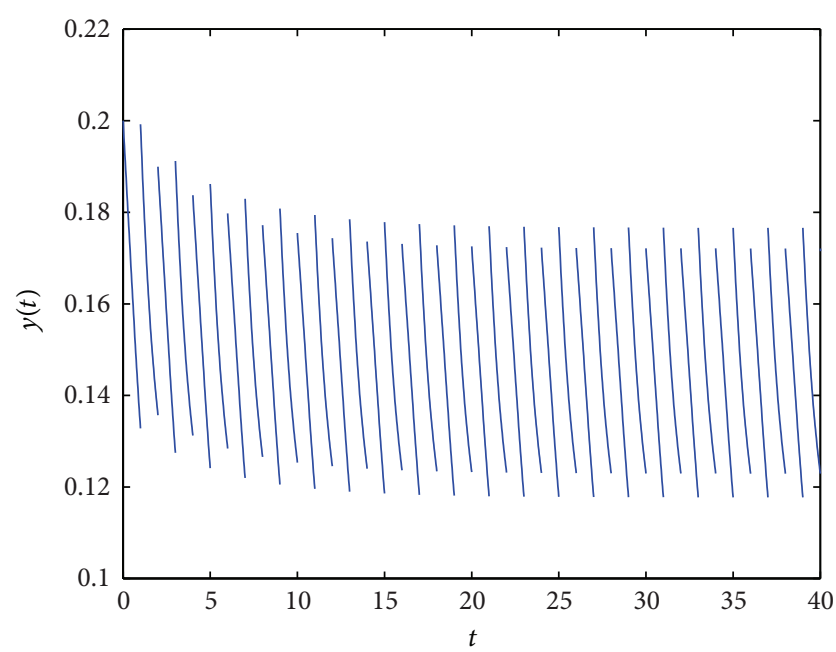

Figure 1: Permanence of system (4a) and (4b) with $y(0)=0.2$, $a(t)=-0.11+0.1 \cos \pi t, b(t)=1.4+0.1 \sin \pi t, c(t)=0.11+$ $0.1 \cos \pi t, d(t)=1.5+\cos \pi t, \gamma_{2 \kappa-1} \equiv 0.5$, and $\gamma_{2 \kappa} \equiv 0.4$. Time series of species $y$ for $t \in[0,40]$.

Thus,

$$
V\left(\tau_{m}^{+}\right) \exp \left(-\psi\left(t-\tau_{m}\right)\right) \longrightarrow 0, \quad \text { as } t \longrightarrow+\infty .
$$

Therefore, from (40) and (42), we obtain that $V(t) \rightarrow 0$ as $t \rightarrow+\infty$; that is,

$$
\left|\ln y^{*}(t)-\ln y(t)\right| \longrightarrow 0, \quad \text { as } t \longrightarrow+\infty .
$$

By the mean value theorem, one has

$$
\lim _{t \rightarrow+\infty}\left|y^{*}(t)-y(t)\right|=0 .
$$

The proof of Theorem 6 is complete.

Remark 7. The positive impulse $p$ has the effect on the global attractivity of system (7a) and (7b).

\section{Discussion}

In this paper, we have proposed a periodic single species model with constant impulsive perturbation which is a continuation of the work of [1]. From systems (4a), (4b), (7a), and (7b), we can see that the equation (4a) or (7a) describes the density variation of species $y$ in a periodically varying environment and the impulsive condition (4b) or (7b) reflects the possible external effects under which the population density changes very rapidly. Result A shows that the suitable large linear impulsive perturbations $\gamma_{k}$ are favorable for the permanence of system (4a) and (4b) while Theorem 4 indicates that the permanence of system (7a) and (7b) is irrespective of the size of the positive constant impulse $p$. As far as the global attractivity is concerned, Result B shows that the suitable large linear impulsive perturbations $\gamma_{k}$ and self-inhibition rate $b(t)$ are favorable for the global attractivity of system (4a) and (4b). Theorem 6 shows that the suitable

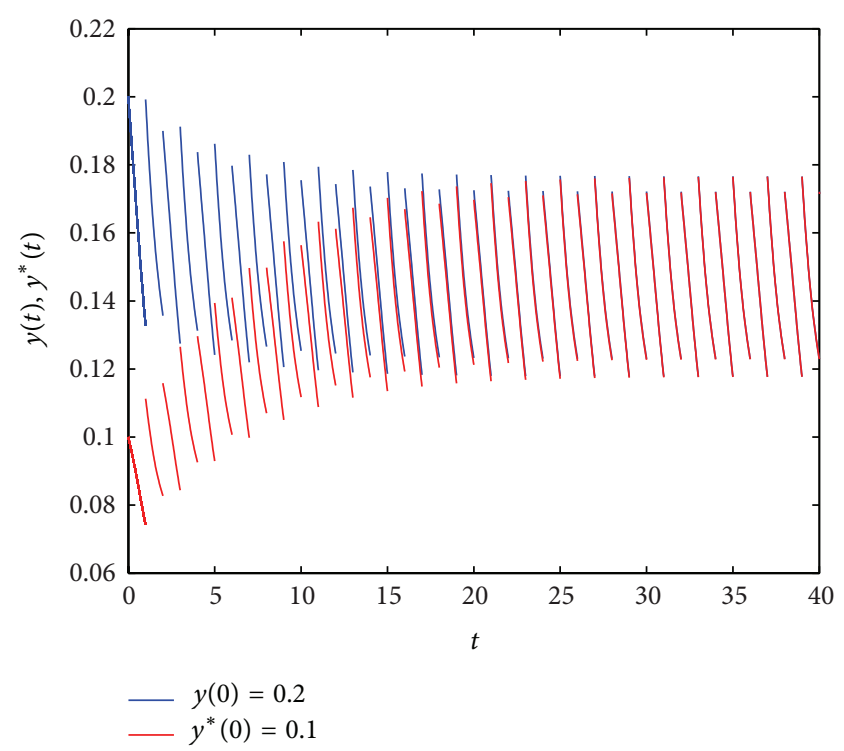

FIGURE 2: Global attractivity of system (4a) and (4b) with $a(t)=$ $-0.11+0.1 \cos \pi t, b(t)=1.4+0.1 \sin \pi t, c(t)=0.11+0.1 \cos \pi t$, $d(t)=1.5+\cos \pi t, \gamma_{2 \kappa-1} \equiv 0.5$, and $\gamma_{2 \kappa} \equiv 0.4$. The positive solution $y^{*}(t)$ with $y^{*}(0)=0.1$ tends to the positive solution $y(t)$ with $y(0)=$ 0.2 .

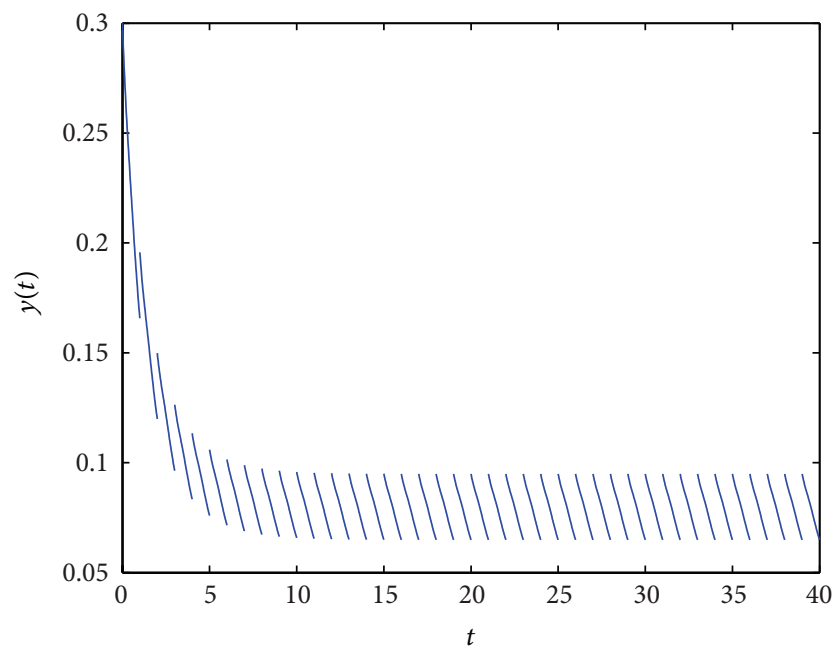

Figure 3: Permanence of system (7a) and (7b) with $y(0)=0.3$, $a(t)=-0.2+0.1 \sin 2 \pi t, b(t)=1.5+0.1 \cos 2 \pi t, c(t)=0.11+$ $0.1 \sin 2 \pi t, d(t)=1.5+\sin 2 \pi t$, and $p=0.03$. Time series of species $y$ for $t \in[0,40]$.

large self-inhibition rate $b(t)$ is also favorable for the global attractivity of system (7a) and (7b); meanwhile, the positive constant impulse $p$ satisfies a harsh condition; that is, $h+p=$ $H$.

To substantiate our analytical results, we will construct some specific examples. We first consider system (4a) and (4b) with $a(t)=-0.11+0.1 \cos \pi t, b(t)=1.4+0.1 \sin \pi t$, $c(t)=0.11+0.1 \cos \pi t$, and $d(t)=1.5+\cos \pi t$. Let $q=$ $2, \tau_{k}=k \in \mathbf{N}$; it is obvious that $T=2$ and $\tau_{k+2}=$ $\tau_{k}+2$. The linear impulse perturbations satisfy $\gamma_{2 \kappa-1} \equiv 0.5$ 


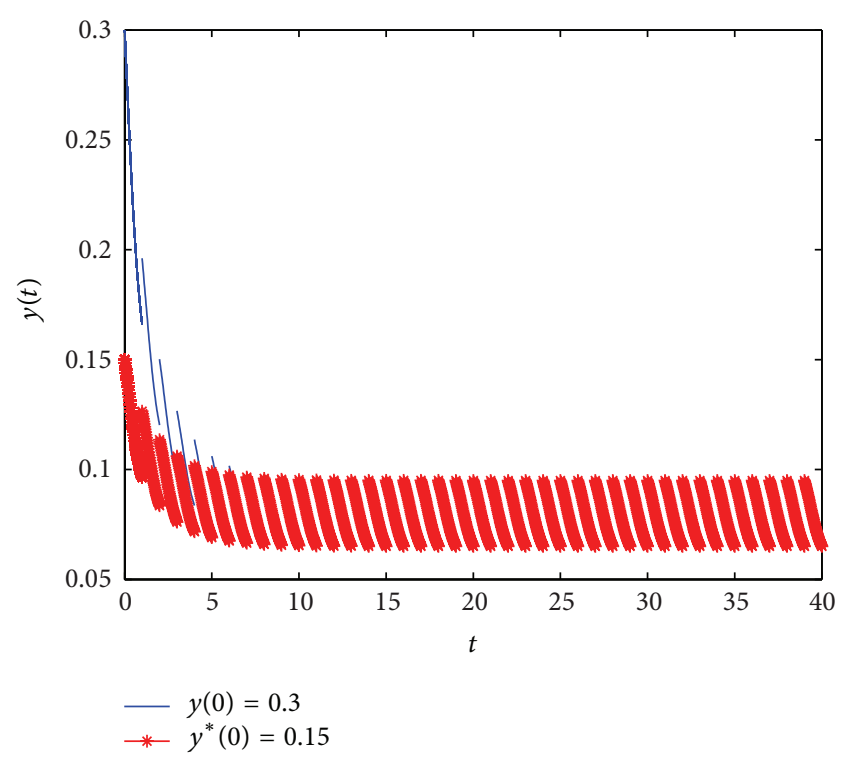

FIgURE 4: Global attractivity of system (7a) and (7b) with $y(0)=0.3$, $a(t)=-0.2+0.1 \cos 2 \pi t, b(t)=1.5+0.1 \sin 2 \pi t, c(t)=0.11+$ $0.1 \cos 2 \pi t, d(t)=1.5+\cos 2 \pi t$, and $p=0.03$. The positive solution $y^{*}(t)$ with $y(0)=0.15$ tends to the positive solution $y(t)$ with $y(0)=$ 0.3 .

(when $k=2 \kappa-1$ ) and $\gamma_{2 \kappa} \equiv 0.4$ (when $k=2 \kappa, k, \kappa \in \mathbf{N}$ ). A simple calculation shows that assumption (5) of Result A holds, and from Figure 1 we may observe that system (4a) and (4b) with $y(0)=0.2$ is permanent (here $m \approx 0.1178$ ). Furthermore, we can verify the validity of Result $B$, and hence system (4a) and (4b) is global attractive. From Figure 2, we observe that the positive solution $y^{*}(t)$ with $y^{*}(0)=0.1$ tends to the positive solution $y(t)$ with $y(0)=0.2$. In the following, we verify the feasibilities of Theorems 4 and 6 . Let us consider system (7a) and (7b) with $a(t)=-0.2+$ $0.1 \sin 2 \pi t, b(t)=1.5+0.1 \cos 2 \pi t, c(t)=0.11+0.1 \sin 2 \pi t$, $d(t)=1.5+\sin 2 \pi t$, and $p=0.03$. Obviously, $T=1$. Let $\tau_{k}=k, k \in \mathbf{N}$; then, $\tau_{k+1}=\tau_{k}+1$. From Figure 3 we see that system (7a) and (7b) with $y(0)=0.3$ is permanent (here $h \approx 0.06487$ ). A calculation shows that assumptions of Theorem 6 are satisfied, and hence system (7a) and (7b) is global attractive (see Figure 4; the positive solution $y^{*}(t)$ with $y^{*}(0)=0.15$ tends to the positive solution $y(t)$ with $y(0)=0.3)$. The above examples imply that the linear and constant impulsive perturbations play important roles and have effect on the above dynamics.

We would like to mention here that some interesting but challenging problems associated with the investigation of system (7a) and (7b) should be the dynamic behaviors if $p$ is replaced with $p\left(\tau_{k}\right)$ or $p\left(\tau_{k}, y\left(\tau_{k}\right)\right)$, or the impulse is random impulses. We leave them for future work.

\section{Conflict of Interests}

The authors declare that there is no conflict of interests regarding the publication of this paper.

\section{Acknowledgments}

The authors would like to thank the referees for their valuable comments and suggestions that greatly improved the presentation of this work. The work is supported by the National Natural Science Foundation of China (no. 11261017), the Key Project of Chinese Ministry of Education (no. 212111), the Key Laboratory of Biological Resources Protection and Utilization of Hubei Province (PKLHB1329), and the Key Subject of Hubei Province (Forestry).

\section{References}

[1] R. Tan, Z. Liu, and R. Cheke, "Periodicity and stability in a single-species model governed by impulsive differential equation," Applied Mathematical Modelling, vol. 36, no. 3, pp. 10851094, 2012.

[2] W. B. Wang, J. H. Shen, and J. J. Nieto, "Permanence and periodic solution of predator-prey system with holling type functional response and impulses," Discrete Dynamics in Nature and Society, vol. 2007, Article ID 81756, 15 pages, 2007.

[3] Z. Liu and R. H. Tan, "Impulsive harvesting and stocking in a Monod-Haldane functional response predator-prey system," Chaos, Solitons \& Fractals, vol. 34, no. 2, pp. 454-464, 2007.

[4] Z. Liu, Y. Chen, Z. He, and J. Wu, "Permanence in a periodic delay logistic system subject to constant impulsive stocking," Mathematical Methods in the Applied Sciences, vol. 33, no. 8, pp. 985-993, 2010

[5] Z. Li, T. Wang, and L. Chen, "Periodic solution of a chemostat model with Beddington-DeAnglis uptake function and impulsive state feedback control," Journal of Theoretical Biology, vol. 261, no. 1, pp. 23-32, 2009.

[6] A. d'Onofrio, "On pulse vaccination strategy in the SIR epidemic model with vertical transmission," Applied Mathematics Letters, vol. 18, no. 7, pp. 729-732, 2005.

[7] R. Shi, X. Jiang, and L. Chen, "A predator-prey model with disease in the prey and two impulses for integrated pest management," Applied Mathematical Modelling, vol. 33, no. 5, pp. 2248-2256, 2009.

[8] A. Lakmeche and O. Arino, "Bifurcation of non trivial periodic solutions of impulsive differential equations arising chemotherapeutic treatment," Dynamics of Continuous, Discrete and Impulsive Systems, vol. 7, no. 2, pp. 165-187, 2000.

[9] Y. Zhang and J. Sun, "Stability of impulsive neural networks with time delays," Physics Letters A, vol. 348, no. 1-2, pp. 44-50, 2005.

[10] A. Anguraj, S. Wu, and A. Vinodkumar, "The existence and exponential stability of semilinear functional differential equations with random impulses under non-uniqueness," Nonlinear Analysis: Theory, Methods \& Applications, vol. 74, no. 2, pp. 331342, 2011.

[11] S. Wu, D. Han, and X. Meng, " $p$-moment stability of stochastic differential equations with jumps," Applied Mathematics and Computation, vol. 152, no. 2, pp. 505-519, 2004.

[12] S. Wu and Y. Duan, "Oscillation, stability, and boundedness of second-order differential systems with random impulses," Computers \& Mathematics with Applications, vol. 49, no. 9-10, pp. 1375-1386, 2005.

[13] J. Shen, Z. Luo, and X. Liu, "Impulsive stabilization of functional-differential equations via Liapunov functionals," Journal of Mathematical Analysis and Applications, vol. 240, no. 1, pp. $1-15,1999$. 
[14] D. Xu, W. Zhu, and S. Long, "Global exponential stability of impulsive integro-differential equation," Nonlinear Analysis: Theory, Methods \& Applications, vol. 64, no. 12, pp. 2805-2816, 2006.

[15] D. Xu and Z. Yang, "Impulsive delay differential inequality and stability of neural networks," Journal of Mathematical Analysis and Applications, vol. 305, no. 1, pp. 107-120, 2005.

[16] H. Chen, "Impulsive-integral inequality and exponential stability for stochastic partial differential equations with delays," Statistics \& Probability Letters, vol. 80, no. 1, pp. 50-56, 2010.

[17] H. Chen, X. Zhang, and Y. Zhao, "The existence and exponential stability for random impulsive integrodifferential equations of neutral type," Advances in Difference Equations, vol. 2010, Article ID 540365, 18 pages, 2010.

[18] D. Ludwig, D. D. Jones, and C. S. Holling, "Qualitative analysis of insect outbreak systems: the spruce budworm and forest," Journal of Animal Ecology, vol. 47, no. 1, pp. 1315-1332, 1978.

[19] J. D. Murray, Mathematical Biology I: An introduction, vol. 17, Springer, Berlin, Germany, 3rd edition, 2002.

[20] V. Lakshmikantham, D. D. Bainov, and P. S. Simeonov, Theroy of Impulsive Differential Equations, vol. 6 of Series in Modern Applied Mathematics, World Scientific Publishing, Singapore, 1989. 


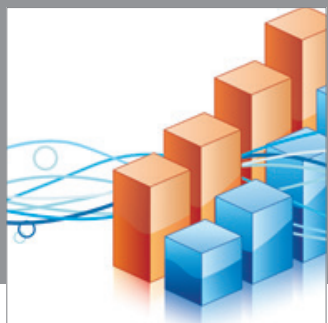

Advances in

Operations Research

mansans

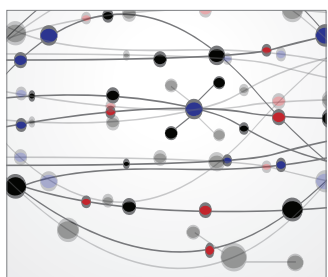

The Scientific World Journal
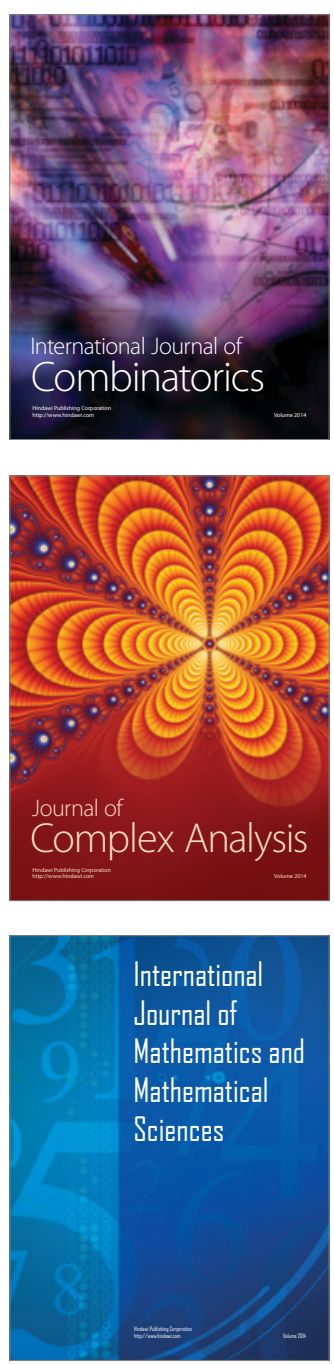
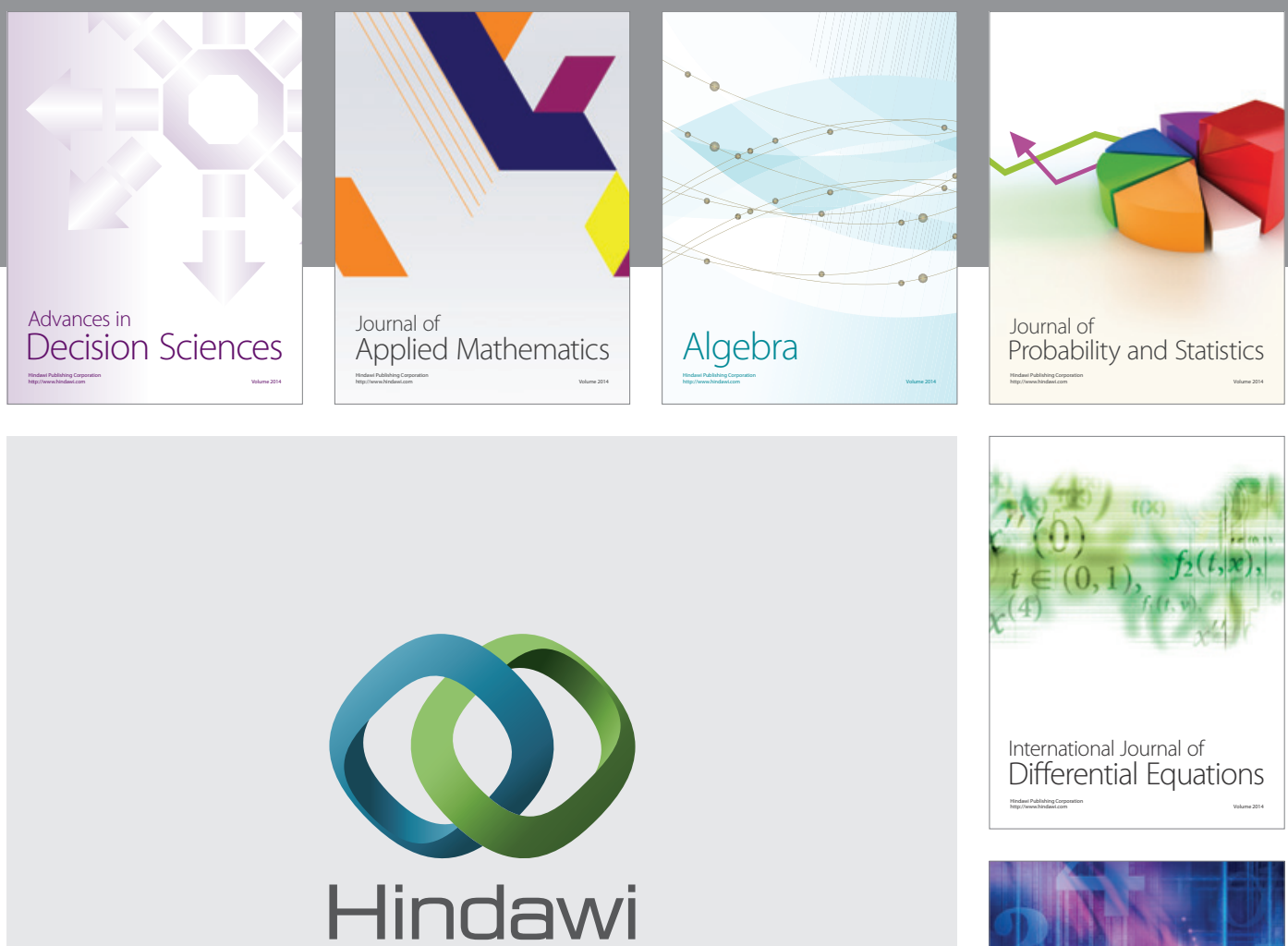

Submit your manuscripts at http://www.hindawi.com
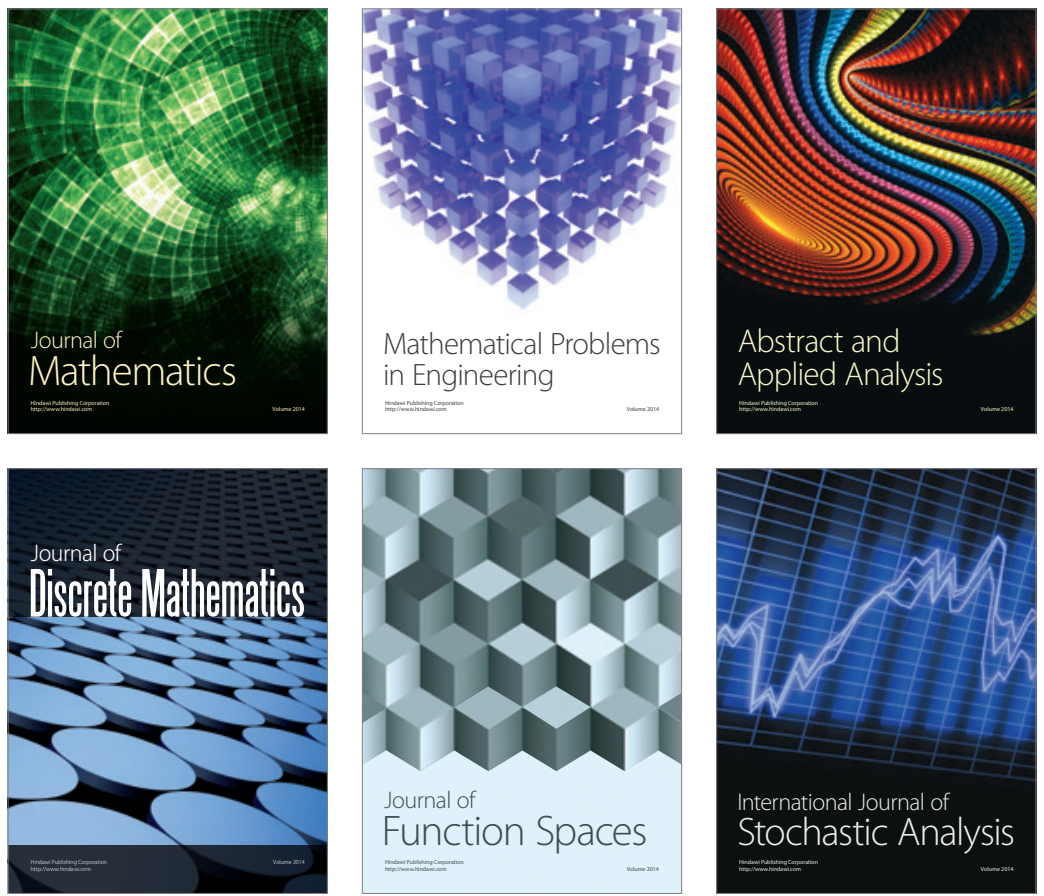

Journal of

Function Spaces

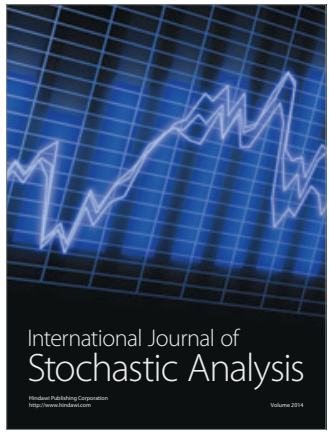

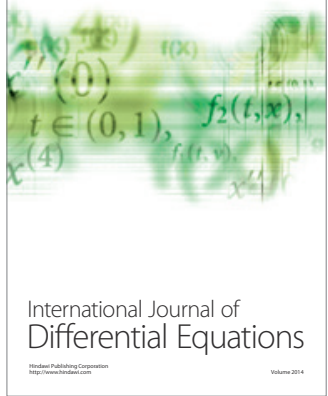
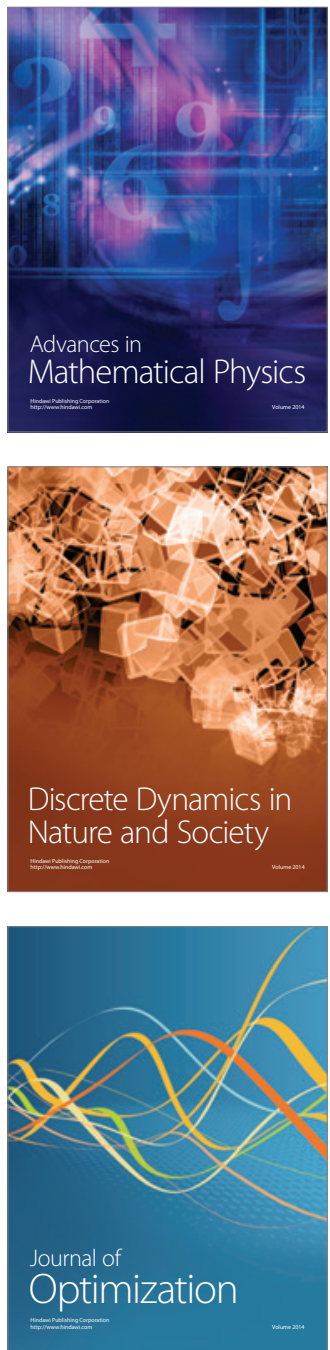